\section{DIGITAL WHOLE SLIDE IMAGE (WSI) SCORING IS EQUIVALENT TO MICROSCOPE GLASS SLIDE SCORING FOR EVALUATION OF PROGRAMMED DEATH-LIGAND 1 (PD-L1) EXPRESSION ACROSS MULTIPLE TUMOR INDICATIONS}

Micki Adams*, Deanna Moquin, Joshua Littrell, Jay Milo, Stephanie Hund, Angeliki Apostolaki. Agilent Technologies, Inc., Santa Barbara, CA, USA

Background The COVID-19 pandemic brought a host of new challenges, including the immediate need for digital solutions addressing the lack of remote options available to pathologists in the field of immunohistochemistry (IHC)-based companion diagnostics for Programmed Death-Ligand 1 (PD-L1) expression evaluation in tumor tissues. Agilent Technologies, Inc. investigated concordance of PD-L1 expression results recorded by trained pathologists between stained glass slides and digital whole slide images (WSIs). Formalin-fixed, paraffin-embedded (FFPE) specimens of eleven tumor indications (table 1) were evaluated in this study. Specimens were stained using the qualitative IHC assay PD-L1 IHC 22C3 pharmDx on Autostainer Link 48 and scored using TPS (Tumor Proportion Score) or CPS (Combined Positive Score) algorithms at six validated cutoffs. ${ }^{1}$ The objective was to demonstrate equivalency between digital WSI and microscope glass slide scoring.

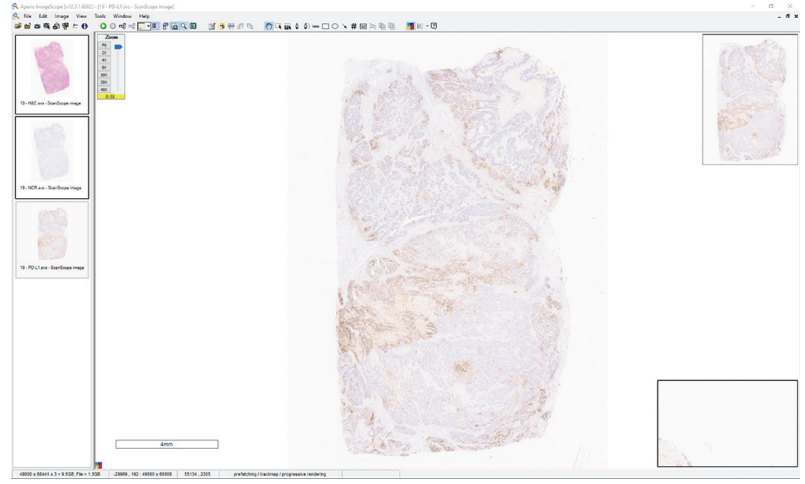

Abstract 36 Figure 1 Digital WSI of a triple-negative breast carcinoma (TNBC) specimen stained with PD-L1 IHC 22C3 pharmDx primary antibody and viewed on Aperio ImageScope software with corresponding H\&E and NCR WSIs for use as aids in the interpretation of PD-L1 staining.*

*Tissue sample supplied by BiolVT (Hicksville, NY, USA)

Abstract 36 Table 1 Algorithm-cutoff pairs tested

\begin{tabular}{|c|c|c|c|}
\hline Algorithm & Cutoff & $\begin{array}{c}\text { \# of Tumor } \\
\text { Indications } \\
\text { Tested }\end{array}$ & $\begin{array}{l}\text { Tumor Indications Tested/ \# of Specimens Tested from Each } \\
\text { Indication }\end{array}$ \\
\hline \multirow[t]{4}{*}{ CPS } & 1 & 8 & $\begin{array}{l}\text { Urothelial carcinoma (UC): } 30 \\
\text { Head and neck squamous cell carcinoma (HNSCC): } 32 \\
\text { Cervical cancer. } 34 \\
\text { Breast carcinoma (BC) including triple-negative breast carcinoma } \\
\text { (TNBC): } 37 \\
\text { Renal cell carcinoma (RCC): } 30 \\
\text { Biliary tract adeno cancer (BTAC): } 32 \\
\text { Small cell lung cancer (SCLC): } 30 \\
\text { Colorectal carcinoma (CRC): } 38\end{array}$ \\
\hline & 10 & 5 & $\begin{array}{l}\text { Esophageal cancer: } 30 \\
\text { UC: } 30 \\
\text { Cervical cancer: } 34 \\
\text { BC (including TNBC): } 30 \\
\text { BTAC: } 32\end{array}$ \\
\hline & 20 & 1 & HNSCC: 122 \\
\hline & 50 & 1 & HNSCC: 122 \\
\hline \multirow[b]{2}{*}{ TPS } & $1 \%$ & 2 & $\begin{array}{l}\text { Non-small cell lung cancer (NSCLC): } 90 \\
\text { NSCLC cytology: } 30\end{array}$ \\
\hline & $50 \%$ & 3 & $\begin{array}{l}\text { HNSCC: } 68 \\
\text { NSCLC: } 40 \\
\text { NSCLC cytology: } 30 \\
\end{array}$ \\
\hline
\end{tabular}

Abstract 36 Table 2 Minimum computer monitor requirements for viewing WSIs on Aperio ImageScope

\begin{tabular}{|l|l|l|}
\hline \multicolumn{1}{|c|}{ Component } & \multicolumn{1}{|c|}{ Aperio Image Hub } & \multicolumn{1}{c|}{$\begin{array}{c}\text { eSlide Manager and Aperio } \\
\text { Image Analysis Workstation }\end{array}$} \\
\hline Display type & LCD (flat panel) & LCD (flat panel) \\
\hline Screen Resolution & $1680(\mathrm{~h}) \times 1050(\mathrm{v})$ pixels & $1680(\mathrm{~h}) \times 1050(\mathrm{v})$ pixels \\
\hline Screen size & 24 -inch & 24 -inch \\
\hline Color depth & $24-$ bit & $24-\mathrm{bit}$ \\
\hline Brightness & $250 \mathrm{~cd} / \mathrm{m}^{2}$ or greater & $250 \mathrm{~cd} / \mathrm{m}^{2}$ or greater \\
\hline Contrast ratio & $500: 1$ & $500: 1$ \\
\hline
\end{tabular}

Abstract 36 Table 3 Glass slide vs. digital WSI NPA/PPA/OA results summary for the six algorithm-cutoff pairs tested

\begin{tabular}{|c|c|c|c|c|c|c|}
\hline \multicolumn{7}{|c|}{ Agreement Summary } \\
\hline \multirow[b]{2}{*}{ Algorithm } & \multirow[b]{2}{*}{ Cutoff } & \multirow[b]{2}{*}{$\begin{array}{c}\text { Total } \\
\text { Comparisons }\end{array}$} & \multirow[b]{2}{*}{$\begin{array}{l}\text { Performance } \\
\text { Criteria }\end{array}$} & \multirow[b]{2}{*}{$\begin{array}{c}\text { Point } \\
\text { Estimate }\end{array}$} & \multicolumn{2}{|c|}{$\begin{array}{c}\text { 95\% Confidence } \\
\text { Interval (Bootstrap) }\end{array}$} \\
\hline & & & & & $\begin{array}{l}\text { Lower- } \\
\text { bound: } \\
2.5 \%\end{array}$ & $\begin{array}{l}\text { Upper- } \\
\text { bound: } \\
97.5 \%\end{array}$ \\
\hline \multirow{3}{*}{ CPS } & \multirow{3}{*}{1} & 358 & NPA & $96.6 \%$ & 94.521 & 98.603 \\
\hline & & 426 & PPA & $97.9 \%$ & 96.487 & 99.061 \\
\hline & & 784 & $O A$ & $97.3 \%$ & 95.802 & 98.599 \\
\hline \multirow{3}{*}{ CPS } & \multirow{3}{*}{10} & 223 & NPA & $93.3 \%$ & 89.091 & 96.847 \\
\hline & & $\frac{250}{243}$ & PPA & $95.1 \%$ & 91.968 & 97.561 \\
\hline & & 466 & $O A$ & $94.2 \%$ & 91.845 & 96.360 \\
\hline \multirow{3}{*}{ CPS } & \multirow{3}{*}{20} & 164 & NPA & $91.5 \%$ & 86.310 & 95.808 \\
\hline & & 197 & PPA & $88.3 \%$ & 83.505 & 92.746 \\
\hline & & 361 & OA & $89.8 \%$ & 85.912 & 93.333 \\
\hline \multirow{3}{*}{ CPS } & \multirow{3}{*}{50} & 179 & NPA & $91.6 \%$ & 87.059 & 95.652 \\
\hline & & 180 & PPA & $90 \%$ & 84.153 & 95.055 \\
\hline & & 359 & $O A$ & $90.8 \%$ & 86.944 & 94.167 \\
\hline \multirow{3}{*}{ TPS } & \multirow{3}{*}{$1 \%$} & 171 & NPA & $90.6 \%$ & 85.965 & 94.767 \\
\hline & & 188 & PPA & $97.3 \%$ & 94.149 & 99.479 \\
\hline & & 359 & $O A$ & $94.2 \%$ & 91.365 & 96.657 \\
\hline \multirow{3}{*}{ TPS } & \multirow{3}{*}{$50 \%$} & 230 & NPA & $91.7 \%$ & 87.611 & 95.575 \\
\hline & & 180 & PPA & $93.1 \%$ & 89617 & 97.340 \\
\hline & & 410 & $O A$ & $92.7 \%$ & 89.731 & 95.400 \\
\hline
\end{tabular}

Abstract 36 Table 4 Number of PD-L1 expression level discordances in HNSCC CPS $\geq 20$ study for predefined negative, near-cutoff (NCO) negative, NCO positive, and positive categories based on specimen screening data assigned by one or more Agilent pathologists prior to the study

\begin{tabular}{|c|c|c|}
\hline \multicolumn{2}{|c|}{ PD-L1 Expression Level Discordances with Respect to Screening Data at CPS $\geq 20$ Cutoff } \\
\hline $\begin{array}{c}\text { Specimen } \\
\text { Category with CPS } \\
\geq 20 \text { Cutoff }\end{array}$ & $\begin{array}{c}\text { \# of Discordances Generated by } \\
\text { Glass Slide Scoring }\end{array}$ & $\begin{array}{c}\text { \# of Discordances Generated by Digital } \\
\text { WSI Scoring }\end{array}$ \\
\hline Negative & 10 & 8 \\
\hline Negative NCO & 15 & 10 \\
\hline Positive NCO & 3 & 6 \\
\hline Positive & 6 & 5 \\
\hline
\end{tabular}

Abstract 36 Table 5 Number of PD-L1 expression level discordances in HNSCC CPS $\geq 50$ study for predefined negative, NCO negative, NCO positive and positive categories based on specimen screening data assigned by one or more Agilent pathologists prior to the study

\begin{tabular}{|c|c|c|}
\hline \multicolumn{2}{|c|}{ PD-L1 Expression Level Discordances with Respect to Screening Data at CPS $\geq 50$ Cutoff } \\
\hline $\begin{array}{c}\text { Specimen } \\
\text { Category with } \\
\text { CPS } \geq 50 \text { Cutoff }\end{array}$ & \# of Discordances Generated by Glass \\
Slide Scoring & $\begin{array}{c}\text { \# of Discordances Generated by } \\
\text { Digital WSI Scoring }\end{array}$ \\
\hline Negative & 2 & 6 \\
\hline Negative NCO & 10 & 9 \\
\hline Positive NCO & 10 & 13 \\
\hline Positive & 2 & 4 \\
\hline
\end{tabular}

Methods Three Agilent-certified pathologists evaluated specimen PD-L1 expression level (positive/negative) using CPS and/ or TPS at relevant cutoff(s) for each indication (table 1) using two scoring modalities for the same specimen sets: 1) light microscope, and, 2) digital monitor (WSI) with a minimum 14-day washout period between glass slide and WSI reads. WSIs were generated using Leica's Aperio AT2 scanner and evaluated using Aperio ImageScope software (figure 1) on appropriate monitors (table 2). Concordance between specimen glass slide (reference condition) and WSI PD-L1 expression results was assessed per cutoff on pooled data from all applicable indications using negative percent agreement (NPA), 
positive percent agreement (PPA) and overall agreement (OA) with 95\% two-sided percentile bootstrap confidence intervals (CI); the acceptance criteria for equivalency at each cutoff were set at CI lower-bounds (CILBs) $\geq 85 \%$. Discordant comparisons with respect to specimen screening data generated prior to inclusion in the study were also analyzed where applicable.

Results NPA/PPA/OA CILBs for the CPS $\geq 1$, CPS $\geq 10$, TPS $\geq 1 \%$, and TPS $\geq 50 \%$ cutoffs were $\geq 85 \%$ (table 3 ). NPA and OA CILBs at CPS $\geq 20$ and CPS $\geq 50$ were $\geq 85 \%$; PPA CILBs were $83.2 \%$ and $84.2 \%$, respectively. Discordant comparisons analysis for CPS $\geq 20$ and CPS $\geq 50$ suggested that WSI is not more prone to discordances in PD-L1 expression level than glass slide scoring when compared to specimen screening data (tables 4 and 5).

Conclusions Glass slide and WSI scoring are equivalent across multiple validated cutoffs and tumor indications tested for PD-L1 expression using PD-L1 IHC 22C3 pharmDx with CPS and/or TPS algorithms and are, thus, considered interchangeable scoring modalities.

Acknowledgements $<\mathrm{i}>$ We would like to thank our colleagues at Agilent Technologies, Inc. and all of the pathologists involved in study specimen scoring for their valuable contributions to this study. Samples/tissue supplied by Conversant Biologics.Tissue samples supplied by BioIVT (Hicksville, NY, USA)The data and biospecimens used in this project were provided by Centre Hospitalier Universitaire (CHU) de Nice (Nice, France), Contract Research Ltd (Charlestown, Nevis), National BioService LLC (Saint Petersburg, Russia), Sofia Bio LLC (New York, NY, USA), US Biolab (Gaithersburg, MD, USA), Nottingham University Hospitals NHS Trust (Nottingham, UK), Gundersen Medical Foundation Center Biobank (La Crosse, WI, USA), LLC Biomedica CRO (Kyiv, Ukraine), Clinfound Clinical Research Services Pvt Ltd (Idukki, Kerala, India), SageBio LLC (Sharon, MA, USA), GLAS (WinstonSalem, NC, USA), Hospices Civils de Lyon (Lyon, France), IOM Ricera (Viagrande, Italy), Clin-Path Diagnostics (Tempe, AZ, USA), Centre Antoine Lacassagne (CAL; Nice, France), CHU de Bordeaux (Biobank ID: BB-0033-00036; Bordeaux, France) and contributions by clinical personnel from Centre de ressources biologiques, and SELARL DIAG (Nice, France) with appropriate ethics approval and through Trans-Hit Biomarkers Inc. Biological materials were provided by the Ontario Tumour Bank, which is supported by the Ontario Institute for Cancer Research (Toronto, Ontario, Canada) through funding provided by the Government of Ontario.Tissue samples were provided by the Cooperative Human Tissue Network which is funded by the National Cancer Institute. Other investigators may have received specimens from the same subjects. $</ \mathrm{i}>$

Trial Registration N/A

\section{REFERENCE}

1. P02893/13 Instructions for Use (IFU) for PD-L1 IHC 22C3 pharmDx Human Cancer (SK00621-4) Package Insert

Ethics Approval N/A

Consent N/A

http://dx.doi.org/10.1136/jitc-2021-SITC2021.036 